Pacific

Journal of

Mathematics

\author{
ON A GALOIS CONNECTION \\ BETWEEN THE SUBFIELD LATTICE \\ AND THE MULTIPLICATIVE SUBGROUP LATTICE
}

JoHN K. MCVEY

Volume 264 No. 1

July 2013 


\title{
ON A GALOIS CONNECTION BETWEEN THE SUBFIELD LATTICE AND THE MULTIPLICATIVE SUBGROUP LATTICE
}

\author{
JOHN K. MCVEY
}

\begin{abstract}
Given finite fields $F<E$, we present a collection of subgroups $C \leq E^{\times}$ and establish, to each $C$, a Galois connection between the intermediate field lattice $\mathscr{E}=\{L \mid F \leq L \leq E\}$ and $C$ 's subgroup lattice. Our main result is that, in all but an extremely limited and completely determined family, the closed subset of $\mathscr{E}$ is $\mathscr{E}$ itself, establishing a natural bijection between $\mathscr{E}$ and the lattice $\{L \cap C \mid L \in \mathscr{E}\}$. As an application, we use this bijection to calculate the set of degrees for the complex-valued irreducible representations of the split extension $C \times \operatorname{Gal}(E / F)$.
\end{abstract}

\section{Introduction}

In $\S 3$ of [McVey 2004], generalizing results in $\S 5$ of [Riedl 1999], we worked towards (among other things) a better understanding of the groups $C \rtimes \operatorname{Gal}(E / F)$ for finite fields $F<E$, where $C<E^{\times}$is the subgroup of order $\left|E^{\times}: F^{\times}\right|$. While working to generalize those results further, we discovered a Galois connection which itself is worthy of further study. This paper's intent is to record the Galois connection as well as the research that motivated its initial study. The primary assertion of the Main Theorem is that, but for a completely determined and rather limited family, the intermediate field lattice $\mathscr{E}=\{L \mid F \leq L \leq E\}$ is itself one of the two closed subsets in the Galois connection, thereby determining a canonical bijection between $\mathscr{E}$ and the other closed set $\{L \cap C \mid L \in \mathscr{E}\}$. As to the motivating research, we use this bijection to calculate the degrees of the irreducible complex representations of the aforementioned split extension $C \rtimes \operatorname{Gal}(E / F)$, showing every integer allowed by Itô's theorem is a degree.

\section{Towards the Galois connection}

Our focus in this paper is on monotone Galois connections. To avoid confusion between monotone and antitone connections, we define the term and present the basic relevant results. Two monotone nondecreasing functions $f: \mathscr{A} \rightarrow \mathscr{B}$ and $g: \mathscr{B} \rightarrow \mathscr{A}$ on partially ordered sets $(\mathscr{A}, \leq)$ and $(\mathscr{B}, \leq)$ form a monotone Galois

MSC2010: primary 06A15; secondary 20C15.

Keywords: Galois correspondence, lattice, character degree, finite field. 
connection if

$$
f(a) \leq b \Longleftrightarrow a \leq g(b)
$$

over all $a \in \mathscr{A}$ and $b \in \mathscr{B}$. The function $f$ is the lower adjoint and $g$ is the upper adjoint. The closed sets $\mathscr{A}_{0}$ and $\mathscr{S}_{0}$ of $\mathscr{A}$ and $\mathscr{B}$ respectively are defined by $\mathscr{A}_{0}=g(\mathscr{B})$ and $\mathscr{B}_{0}=f(\mathscr{A})$, and satisfy

$$
\begin{aligned}
& \mathscr{A}_{0}=\{a \in \mathscr{A} \mid g \circ f(a)=a\}=g \circ f(\mathscr{A}), \\
& \mathscr{B}_{0}=\{b \in \mathscr{B} \mid f \circ g(b)=b\}=f \circ g(\mathscr{B}) .
\end{aligned}
$$

The functions $f$ and $g$ are inverse bijections between the sets $\mathscr{A}_{0}$ and $\mathscr{B}_{0}$.

Turning now specifically to our setting of finite fields $F<E$, label by $\pi$ the set of primes which divide $\left|F^{\times}\right|=|F|-1$. The collection of groups to which the Galois connection applies consists of all subgroups $C \leq E^{\times}$for which the index $\left|E^{\times}: C\right|$ is a $\pi$-number (thus naturally generalizing results in [Riedl 1999] where hypotheses guaranteed $\left.\left|E^{\times}: C\right|=\left|F^{\times}\right|\right)$. Fixing a group $C$, the upper adjoint is very easy to describe; it is the function "intersect with $C$ ".

As to the lower adjoint, define the $F$-closure $\hat{X}$ of a subset $X \subseteq E$ to be the smallest subfield of $E$ which contains $X \cup F$. In other words, $\hat{X}$ is the intersection of all fields $L$ satisfying $X \cup F \subseteq L \leq E$. It should be obvious that $F$-closure actually is a closure operator (i.e.,

$$
\hat{X} \supseteq X \quad \text { and } \quad \hat{\hat{X}}=\hat{X}
$$

over all subsets $X \subseteq E)$, and that a Galois automorphism $\sigma \in \operatorname{Gal}(E / F)$ centralizes $X$ if and only if it centralizes $\hat{X}$. The partially ordered sets in our Galois connection are the lattices

$$
\mathscr{E}=\{L \mid F \leq L \leq E \text { is a field }\} \text { and } \mathscr{C}=\{D \mid D \leq C \text { is a group }\},
$$

ordered by inclusion. The functions $X \mapsto X \cap C$ and $X \mapsto \hat{X}$ are obviously monotone. Given $D \in \mathscr{C}$ and $L \in \mathscr{E}$, and noting that $L \cap C=L^{\times} \cap C \in \mathscr{C}$, we have

$$
\widehat{D} \subseteq L \Longleftrightarrow D \subseteq L \Longleftrightarrow D \subseteq L \cap C,
$$

showing that $\hat{\cdot}$ is a lower adjoint while $(\cdot) \cap C$ is an upper adjoint. Therefore, as $\mathscr{A}_{0}=g(\mathscr{B})$, the closed subset of $\mathscr{C}$ is $\mathscr{C}_{0}=\{L \cap C \mid L \in \mathscr{E}\}$.

We are now ready to state the Main Theorem. All but the last two sentences were proven in the above discussion. Those last two sentences are the true content of the theorem, and their proof is at the end of this section.

Main Theorem. Let $F<E$ be finite fields and label by $\pi$ the set of primes dividing $|F|-1$. Let $C$ be a subgroup of $E^{\times}$whose index $\left|E^{\times}: C\right|$ is a $\pi$-number. Given the partially ordered sets defined by (1), the functions $\hat{\cdot}: \mathscr{C} \rightarrow \mathscr{E}$ and $(\cdot) \cap C: \mathscr{E} \rightarrow \mathscr{C}$ 
are respectively the lower and upper adjoints of a monotone Galois connection, and thus provide inverse bijections between the closed subsets $\mathscr{C}_{0} \subseteq \mathscr{C}$ and $\mathscr{E}_{0} \subseteq \mathscr{E}$. The closed subset $\mathscr{C}_{0}$ of $\mathscr{C}$ is the lattice $\mathscr{C}_{0}=\{L \cap C \mid L \in \mathscr{E}\}$. If $|F|$ is a Mersenne prime, $|E: F|$ is even, and 4 does not divide $|C|$, then the closed subset $\mathscr{E}_{0}$ of $\mathscr{E}$ is the set $\mathscr{E}_{0}=\mathscr{E} \backslash\{K\}$ where $|K: F|=2$. Otherwise, $\mathscr{E}=\mathscr{E}_{0}$.

Our argument for the as yet unproven portion of the Main Theorem relies fundamentally on number theory. We ask the reader to recall Zsigmondy's prime theorem, as it is the foundation for what follows.

Theorem 1 [Zsigmondy 1892]. Let $a, b, n$ be positive integers and assume $a, b$ are coprime and not both 1 . Then, $a^{n}-b^{n}$ has a prime divisor which does not divide $a^{k}-b^{k}$ for integers $0<k<n$, except when either

$$
n=6 \text { and }\{a, b\}=\{1,2\} \quad \text { or } n=2 \text { and } a+b \text { is } a \text { 2-power. }
$$

Aside from specifying $\{a, b\}$ as $\{q, 1\}$ with $q$ a prime-power, the main point behind Corollary 2 is that the order of the quantifiers changed (from ' $\exists$ prime $\forall k$ ' in Zsigmondy's theorem to ' $\forall k \exists$ prime' in the corollary).

Corollary 2. Let $n>1$ be an integer and $q$ a power of a prime. For each integer $k$ with $0<k<n$, there is a prime which divides $q^{n}-1$ and not $q^{k}-1$, except when $q$ is a Mersenne prime and $n=2$. Conversely, when $q$ is a Mersenne prime, every prime dividing $q^{2}-1$ divides $q-1$.

Proof. As stated previously, Zsigmondy's theorem provides a universal prime (over all $k$ ) unless we are in one of the exceptional cases. First, assume $n=6$ and $q=2$, in which case $q^{n}-1=2^{6}-1=63=3^{2} \cdot 7$. It suffices to check that 3 divides none of $1=2^{1}-1,7=2^{3}-1$, and $31=2^{5}-1$, and that 7 divides neither $3=2^{2}-1$ nor $15=2^{4}-1$.

In the other exceptional case, $n=2$ and $q+1$ is a 2-power. However, Catalan's conjecture (proven in [Mihăilescu 2004]) says that the integer equation $x^{a}-y^{b}=1$ with $a, b>1$ only has the solution $3^{2}-2^{3}$. Because $q+1$ is a 2 -power already, $q$ itself must be prime, hence a Mersenne prime.

As to the converse, when $q$ is a Mersenne prime, the only prime dividing $q+1$ is 2 , which necessarily divides $(q+1)-2=q-1$. As $q^{2}-1=(q+1)(q-1)$, the result follows.

We now leave number theory and move to algebra proper. Our first algebraic goal is a lemma which shows how the number theory embedded in the previous corollary can be applied to finite fields.

Lemma 3. Let $F \leq K \leq L \leq E$ be finite fields. For the set $\pi$ of prime divisors of $\left|F^{\times}\right|$, let $C$ be a subgroup of $E^{\times}$whose index is a $\pi$-number. If the prime $p$ divides $\left|L^{\times}\right|$and not $\left|K^{\times}\right|$, then $p$ divides $|L \cap C: K \cap C|$. 
Proof. The following picture provides insight into this proof.

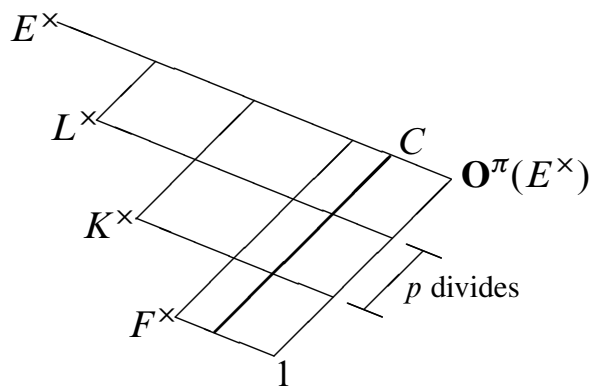

That $p$ does not divide $\left|K^{\times}\right|$implies $p$ does not divide $\left|F^{\times}\right|=q-1$. Since

$$
\left|L^{\times}: L \cap C\right|=\left|L^{\times} C: C\right| \text { divides }\left|E^{\times}: C\right|,
$$

which is a $\pi$-number and thus coprime to $p$, necessarily $p$ divides $|L \cap C|$. As $p$ does not divide $\left|K^{\times}\right|$, it also does not divide $|K \cap C|$.

Theorem 4. Let $q$ be a prime-power, $e>1$ an integer, and $\pi$ the set of primes dividing $q-1$. Label $F=\mathbb{F}_{q}$ and $E=\mathbb{F}_{q^{e}}$, and let $C$ be a subgroup of $E^{\times}$ whose index $\left|E^{\times}: C\right|$ is a $\pi$-number. Then, for all fields $F \leq L \leq E$, the equality $L=\widehat{L \cap C}$ holds, except when the following conditions are all satisfied.

(1) $q$ is a Mersenne prime.

(2) e is even.

(3) $L=\mathbb{F}_{q^{2}}$.

(4) 4 does not divide $|C|$.

When these simultaneously hold, $L \cap C=F \cap C$, so $\widehat{L \cap C}=F<L$.

Proof. Fix the field $L$. Obviously, the set $L \cap C$ is a subset both of $\widehat{L \cap C}$ and of $C$. Therefore,

$$
L \cap C \subseteq \widehat{L \cap C} \cap C \subseteq \widehat{L} \cap C=L \cap C,
$$

and we have equality throughout. Applying (the contrapositive of) Lemma 3 to $K=\widehat{L \cap C}$, the equality $K \cap C=L \cap C$ shows that every prime dividing $\left|L^{\times}\right|$ divides $\left|K^{\times}\right|$. Labelling $\left|L^{\times}\right|=q^{n}-1$ and $\left|K^{\times}\right|=q^{k}-1$, either $k=n$ (and we are done) or we are in the exceptional case of Corollary 2.

Henceforth, assume $n=2$ and $q$ is a Mersenne prime. As $e$ is a multiple of $n$, it is even. Write $q=2^{p}-1$. Consequently,

- $\left|F^{\times}\right|=q-1=2\left(2^{p-1}-1\right)$, which has 2-part exactly 2 , while

- $\left|L^{\times}\right|=q^{2}-1=2^{p}\left|F^{\times}\right|$, which has 2-part $2^{p+1}$.

In particular, the $2^{\prime}$-part of $\left|L^{\times}\right|$is exactly the $2^{\prime}$-part of $\left|F^{\times}\right|$. 


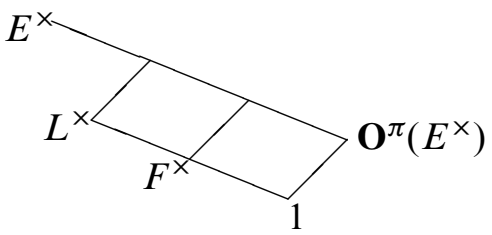

We now split the argument as to whether or not 4 divides $|C|$. If 4 divides $|C|$, then because 4 also divides $\left|L^{\times}\right|$, it divides $|L \cap C|$. However, 4 does not divide $\left|F^{\times}\right|$, so $L \cap C \not F^{\times}$. Accordingly, $F<\widehat{L \cap C} \leq L$, and $F=\mathbb{F}_{q}$ being a maximal subfield of $L=\mathbb{F}_{q^{2}}$ shows $L=\widehat{L \cap C}$.

When 4 does not divide $|C|$, the 2-part of $|L \cap C|$ divides $\left|F^{\times}\right|$. Generally, the $2^{\prime}$-part of $|L \cap C|$ divides $\left|L^{\times}\right|_{2^{\prime}}=\left|F^{\times}\right|_{2^{\prime}}$. It follows that $|L \cap C|$ divides $\left|F^{\times}\right|$. Since $E^{\times}$is cyclic, this shows $L \cap C \leq F^{\times}$, so $L \cap C=F \cap C$.

With the above result in place, we use that $\mathscr{B}_{0}=f \circ g(\mathscr{B})$ to conclude

$$
\mathscr{E}_{0}=\{\widehat{L \cap C} \mid L \in \mathscr{E}\} .
$$

Meanwhile, the $F$-closure $\widehat{L \cap C}$ equals $L$ but for the one exception $\mathbb{F}_{q^{2}}$ when $q$ is Mersenne, $e$ is even, and 4 fails to divide $|C|$. This finishes the proof of the Main Theorem.

\section{Application to degrees}

Our concluding section presents the computations for the character degree set of the split extension $C \rtimes \operatorname{Gal}(E / F)$ when $\mathscr{E}=\mathscr{E}_{0}$. We emphasize once more that this result was the principal impetus for our study of this Galois connection. All standard notations and conventions regarding character theory are taken from [Isaacs 1976]. The following generalizes Theorem 3.2 in [McVey 2004], and the proof here is fundamentally the same as is presented there, the main modification being the use of Theorem 4.

Theorem 5. Fix a prime-power $q$ and an exponent $1<e \in \mathbb{Z}$, and label by $F$ the field $\mathbb{F}_{q}$, by $E$ the field $\mathbb{F}_{q^{e}}$, and by $\pi$ the set of primes dividing $q-1$. Let $\Gamma=\operatorname{Gal}(E / F)$, and fix $C \leq E^{\times}$under the assumption $\left|E^{\times}: C\right|$ is a $\pi$-number. If $q$ is Mersenne and e is even, assume 4 divides $|C|$. Then, $\Gamma$ normalizes $C$ and

$$
\operatorname{cd}(C \Gamma)=\{n \mid n \text { divides } e\} \text {. }
$$

Proof. Because $E^{\times}$is cyclic, every subgroup is characteristic. In particular, $C$ is fixed (setwise) under every field automorphism of $E$, so $\Gamma$ normalizes $C$. As $C$ is cyclic, $\operatorname{Irr}(C)$ contains only linear characters and forms a cyclic group under multiplication. Let $\lambda \in \operatorname{Irr}(C)$ be a generator, noting $\lambda$ is both faithful and a homomorphism. In summary, $\lambda\left(d_{1}\right)=\lambda\left(d_{2}\right)$ implies $d_{1}=d_{2}, \lambda^{m}(d)=\lambda\left(d^{m}\right)$, 
and $\lambda^{\tau}\left(d^{\tau}\right)=\lambda(d)$ for all $d, d_{1}, d_{2} \in C, m \in \mathbb{Z}$, and $\tau \in \Gamma$. Recalling $C \triangleleft C \Gamma$ is abelian, Itô's theorem says every degree in $\operatorname{cd}(C \Gamma)$ divides $|C \Gamma: C|=|\Gamma|=e$.

Conversely, fix a divisor $n$ of $e$, and we will demonstrate an irreducible character of $C \Gamma$ whose degree is $n$. Let $\sigma$ be a generator of $\Gamma$, and label $\Phi=\left\langle\sigma^{n}\right\rangle$, observing that $|\Gamma: \Phi|=n$. Let $L$ be the fixed field for $\sigma^{n}$ in the (usual) Galois correspondence for $E$ over $F$. Hence, $\Phi=\operatorname{Gal}(E / L)$ and $\sigma^{n}$ fixes the subgroup $L \cap C$ of $C$. For some generator $c \in C$, let $L \cap C=\left\langle c^{m}\right\rangle$.

We claim the stabilizer of $\lambda^{m}$ in $\Gamma$ is $\Phi$. Given the claim, the stabilizer of $\lambda^{m}$ in $C \Gamma$ is $C \Phi$, and $\lambda^{m}$ extends to a character $\varphi \in \operatorname{Irr}(C \Phi)$ through for example Corollary 11.22 in [Isaacs 1976]. Also, $\varphi$ induces irreducibly to $C \Gamma$ by Clifford correspondence. Therefore,

$$
n=|\Gamma: \Phi|=|C \Gamma: C \Phi|=|C \Gamma: C \Phi| \varphi(1)=\varphi^{C \Gamma}(1) \in \operatorname{cd}(C \Gamma) .
$$

As $n$ was an arbitrary divisor of $e$, we will have shown the result.

Given $\tau \in \Gamma$ and recalling $\lambda$ is faithful, the equalities

$$
\left(\lambda^{m}\right)^{\tau}(d)=\lambda^{m}\left(d^{\tau^{-1}}\right)=\lambda\left(\left(d^{\tau^{-1}}\right)^{m}\right)=\lambda\left(\left(d^{m}\right)^{\tau^{-1}}\right)
$$

and

$$
\lambda^{m}(d)=\lambda\left(d^{m}\right)
$$

imply that $\tau$ centralizes $\lambda^{m}$ (the left ends are equal) if and only if $\tau^{-1}$ centralizes $d^{m}$ for every $d \in C$ (the right ends are equal). The latter happens exactly when $\tau^{-1}$ centralizes $\left\langle c^{m}\right\rangle=L \cap C$, which occurs if and only if $\tau^{-1}$ centralizes $\widehat{L \cap C}$. As $L=\widehat{L \cap C}$ (Theorem 4), this is equivalent to $\tau^{-1} \in \operatorname{Gal}(E / L)=\Phi$.

In closing, we would be remiss in not mentioning an application of Theorem 5 to a remark made in [Lewis 2001]. For the subsequent, we use the notation of [Lewis 2001]. In the paragraph preceding Lemma 3.4, Dr. Lewis made the comment that "... every divisor of $m$ occurs in $\operatorname{cd}(G / V)$ ", but that particular conclusion was superfluous to Lemma 3.4, so it went unproven. Reading through the first two and a half paragraphs of that proof, $V$ can be viewed as the additive group of the field $\mathbb{F}_{q^{m}}, K / Z$ acts on $\mathbb{F}_{q^{m}}$ by multiplication as if it were a subgroup of $\mathbb{F}_{q^{m}}^{\times}$, and the quotient $H / K$ behaves as a Galois group. Lastly, the hypotheses to Example 2.4 imply $m$ is coprime to $\left(q^{m}-1\right) /(q-1)$. Hence, our result applies to the group $H / Z$, and Lewis' claim about the degrees is an immediate corollary of Theorem 5 and the relations $G / V \cong H$ and $\operatorname{cd}(H)=\operatorname{cd}(H / Z)$.

\section{References}

[Isaacs 1976] I. M. Isaacs, Character theory of finite groups, Pure and Applied Mathematics 69, Academic Press, New York, 1976. MR 57 \#417 Zbl 0337.20005

[Lewis 2001] M. L. Lewis, "Solvable groups whose degree graphs have two connected components", J. Group Theory 4:3 (2001), 255-275. MR 2002g:20015 Zbl 0998.20009 
[McVey 2004] J. K. McVey, "Prime divisibility among degrees of solvable groups", Comm. Algebra 32:9 (2004), 3391-3402. MR 2005i:20012 Zbl 1077.20009

[Mihăilescu 2004] P. Mihăilescu, "Primary cyclotomic units and a proof of Catalan's conjecture", J. Reine Angew. Math. 572 (2004), 167-195. MR 2005f:11051 Zbl 1067.11017

[Riedl 1999] J. M. Riedl, "Character degrees, class sizes, and normal subgroups of a certain class of p-groups”, J. Algebra 218:1 (1999), 190-215. MR 2000f:20025 Zbl 0932.20015

[Zsigmondy 1892] K. Zsigmondy, "Zur Theorie der Potenzreste", Monatsh. Math. Phys. 3:1 (1892), 265-284. MR 1546236 JFM 24.0176.02

Received June 2, 2012. Revised December 10, 2012.

JOHN K. MCVEY

DEPARTMENT OF MATHEMATICS

KENT STATE UNIVERSITY

MATHEMATICS AND COMPUTER SCIENCE BUILding 233

SUMMIT STREET

KENT, OH 44242

UNITED STATES

jmcvey@math.kent.edu 


\title{
PACIFIC JOURNAL OF MATHEMATICS
}

\author{
msp.org/pjm
}

Founded in 1951 by E. F. Beckenbach (1906-1982) and F. Wolf (1904-1989)

\section{EDITORS}

V. S. Varadarajan (Managing Editor)

Department of Mathematics

University of California

Los Angeles, CA 90095-1555

pacific@math.ucla.edu

Paul Balmer

Department of Mathematics

University of California

Los Angeles, CA 90095-1555

balmer@math.ucla.edu

Daryl Cooper

Department of Mathematics

University of California

Santa Barbara, CA 93106-3080 cooper@math.ucsb.edu

Jiang-Hua $\mathrm{Lu}$

Department of Mathematics

The University of Hong Kong

Pokfulam Rd., Hong Kong jhlu@maths.hku.hk
Don Blasius

Department of Mathematics University of California

Los Angeles, CA 90095-1555

blasius@math.ucla.edu

Robert Finn

Department of Mathematics Stanford University

Stanford, CA 94305-2125

finn@math.stanford.edu

Sorin Popa

Department of Mathematics

University of California

Los Angeles, CA 90095-1555

popa@math.ucla.edu

Paul Yang

Department of Mathematics

Princeton University

Princeton NJ 08544-1000

yang@math.princeton.edu

\section{PRODUCTION}

Silvio Levy, Scientific Editor, production@msp.org

\section{SUPPORTING INSTITUTIONS}

ACADEMIA SINICA, TAIPEI

CALIFORNIA INST. OF TECHNOLOGY

INST. DE MATEMÁTICA PURA E APLICADA

KEIO UNIVERSITY

MATH. SCIENCES RESEARCH INSTITUTE

NEW MEXICO STATE UNIV.

OREGON STATE UNIV.

\author{
STANFORD UNIVERSITY \\ UNIV. OF BRITISH COLUMBIA \\ UNIV. OF CALIFORNIA, BERKELEY \\ UNIV. OF CALIFORNIA, DAVIS \\ UNIV. OF CALIFORNIA, LOS ANGELES \\ UNIV. OF CALIFORNIA, RIVERSIDE \\ UNIV. OF CALIFORNIA, SAN DIEGO \\ UNIV. OF CALIF., SANTA BARBARA
}

\author{
Vyjayanthi Chari \\ Department of Mathematics \\ University of California \\ Riverside, CA 92521-0135 \\ chari@math.ucr.edu \\ Kefeng Liu \\ Department of Mathematics \\ University of California \\ Los Angeles, CA 90095-1555 \\ liu@math.ucla.edu \\ Jie Qing \\ Department of Mathematics \\ University of California \\ Santa Cruz, CA 95064 \\ qing@cats.ucsc.edu
}

These supporting institutions contribute to the cost of publication of this Journal, but they are not owners or publishers and have no responsibility for its contents or policies.

See inside back cover or msp.org/pjm for submission instructions.

The subscription price for 2013 is US \$400/year for the electronic version, and \$485/year for print and electronic.

Subscriptions, requests for back issues and changes of subscribers address should be sent to Pacific Journal of Mathematics, P.O. Box 4163, Berkeley, CA 94704-0163, U.S.A. The Pacific Journal of Mathematics is indexed by Mathematical Reviews, Zentralblatt MATH, PASCAL CNRS Index, Referativnyi Zhurnal, Current Mathematical Publications and the Science Citation Index.

The Pacific Journal of Mathematics (ISSN 0030-8730) at the University of California, c/o Department of Mathematics, 798 Evans Hall \#3840, Berkeley, CA 94720-3840, is published monthly except July and August. Periodical rate postage paid at Berkeley, CA 94704, and additional mailing offices. POSTMASTER: send address changes to Pacific Journal of Mathematics, P.O. Box 4163, Berkeley, CA 94704-0163.

PJM peer review and production are managed by EditFLOW ${ }^{\circledR}$ from Mathematical Sciences Publishers.

PUBLISHED BY

mathematical sciences publishers

nonprofit scientific publishing

http://msp.org/

(C) 2013 Mathematical Sciences Publishers 


\section{PACIFIC JOURNAL OF MATHEMATICS}

Volume $264 \quad$ No. $1 \quad$ July 2013

On the center of fusion categories

ALAIN BRUGUIÈRES and ALEXIS VIRELIZIER

Connected quandles associated with pointed abelian groups

W. EdWin Clark, MoHamed ElHamdadi, Xiang-DONG HoU,

MASAHICO SAITO and TIMOTHY YEATMAN

Entropy and lowest eigenvalue on evolving manifolds

HongXin GuO, ROBERT PHILIPOWSKI and ANTON THALMAIER

Poles of certain residual Eisenstein series of classical groups

DIHUA JIANG, BAIYING LIU and LEI ZHANG

Harmonic maps on domains with piecewise Lipschitz continuous metrics 125

HAIGANG LI and CHANGYOU WANG

$q$-hypergeometric double sums as mock theta functions

JEREMY LOVEJOY and ROBERT OSBURN

Monic representations and Gorenstein-projective modules

XIU-HuA LUO and PU ZHANG

Helicoidal flat surfaces in hyperbolic 3-space

Antonio Martínez, JoÃo Paulo dos Santos and Keti

TENENBLAT

On a Galois connection between the subfield lattice and the multiplicative subgroup lattice

JOHN K. MCVEY

Some characterizations of Campanato spaces via commutators on Morrey 221 spaces

Shaoguang Shi and Shanzhen Lu

The Siegel-Weil formula for unitary groups 